\title{
Design, Implementation and Comparison of Low- Cost Laser Scanning Systems for 3D Modeling
}

\author{
Tuba Kurban \\ Dept. of Geomatics Engineering \\ Erciyes University \\ Kayseri, Turkey \\ tubac@erciyes.edu.tr \\ Erkan Besdok \\ Dept. of Geomatics Engineering \\ Erciyes University \\ Kayseri, Turkey \\ ebesdok@erciyes.edu.tr
}

\begin{abstract}
D) modeling of an object or an environment using point clouds is an important problem in many scientific fields such as photogrammetry, remote sensing, materials processing, reverse engineering, construction industry, virtual reality and medicine etc. Laser scanning is an effective technique that facilitates 3D modeling process with providing large amount of 3D point cloud data in a short time. In this study, design process of point laser sensor and line laser sensor based low cost scanner systems is proposed. Performed 3D data measurements with these two different laser scanners show that; point laser range sensor based scanner, that can capture lesser 3D point for per second, provides more detailed and more sensitive measurements. It can be preferred in applications when the details are very important and are suitable for modeling small objects. However, line laser range sensor based scanner can capture much more 3D point data per second and it is suitable for applications where time critical models with large objects and environment.
\end{abstract}

Keywords_laser scanning; 3d data acquisition; point cloud; 3d modeling

techniques, laser scanners provide large amount of 3D data more precisely and quickly [20].

\section{INTRODUCTION}

Laser scanners can obtain large amounts of data called 3D point cloud and this data is used for constructing a 3D model which is used to facilitate the analysis of a real world object or an environment $[1,2]$. 3D scanning technology is used variety of fields such as photogrammetry and remote sensing [3], materials processing and manufacturing [4-8], reverse engineering [5-8], civil engineering [9], virtual reality and augmented reality [10], cultural heritage $[11,12]$ and medical [13] applications.

Wide variety of hardware and software based solutions for 3D data acquisition is commercially available. Laser scanner [14], structured light scanner [15], stereo vision [16], photogrammetry [17], interferometry [18] and shape from shading [19] are popular techniques have been developed. With the advances in technology, accuracy of these devices is increased while costs are decreased. This situation makes 3D data attractive for many applications. Among all these

In this study, two different scanner systems are designed that differs in terms of used laser range sensor type. Point laser and line laser range sensors are mounted on a highly precise pan tilt unit. Coordination, between pan tilt unit that performed the localization and laser range sensors, is controlled precisely by developed 3D data acquisition software.

At the rest of this paper proposed point laser based scanner and line laser based scanner are discussed in section 2 . Comparison of the two different laser range sensor and measurement results are given in section 3. Conclusions are in section 4.

\section{PROPOSED LASER SCANNING MODELS}

Proposed low cost scanning systems consist of a pan tilt unit and two different laser range sensors. Pan tilt unit that used in our system is PTU D46-70 [21] obtained from Directed Perception. PTU-D46-70 can be controlled from a 
computer via RS-232 port. The operation speed of this unit is $60^{\circ} / \mathrm{sec}$ and resolution is $0.012857^{\circ}$ per pan and tilt step. Load capacity is over $4.08 \mathrm{~kg}$. Therefore, it is preferred in many applications such as robotics, computer vision, security, surveillance, industrial automation, tracking, webcams and laser ranging. PTU-D46-70 pan tilt unit is given in Fig. 1.

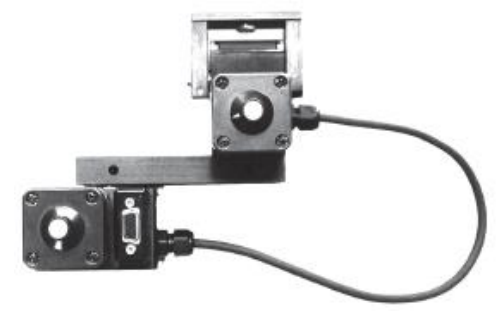

Fig. 1. PTU D46-70 pan tilt unit

Our measurement systems differ in terms of laser scanner sensor types. First laser range sensor that used in our system is FLS C-10 [22] point-laser scanner obtained from Dimetix. Point laser range sensor only detects the distance of one point in its direction of view. The other sensor that used in the system is UTM 30 - LX [23] obtained from Hokuyo. The sensor view direction can be changed by using a rotating mirror. Thus, scanning process performed along a line.

In our measurement system, the pan-tilt system has been placed on a tripod. Each laser range sensor can be mounted on the pan tilt unit. Experimental setup can be seen in Fig. 2. Laser sensors measures continuously while pan tilt unit take steps along maximum and minimum pan and tilt range of motion. PTU-D46-70 pan-tilt has 24836 steps for pan and 5823 steps for tilt. In other words, it can scan $-159.65^{\circ}$ to $159.67^{\circ}$ for pan and $-45.1^{\circ}$ to $29.76^{\circ}$ for tilt. Both pan tilt unit and laser range finder are connected to a host computer and can be controlled from RS-232 port. The details of the proposed models are given in the following sub-sections:

\section{A. Point-laser based scanner}

FLS C-10 is a powerful laser range sensor for industrial applications. It allows accurate and contactless distance measurement over a wide range using the reflection of a laser beam. It measures from 0.05 to 65 meters with 1.0 millimeter accuracy. The laser distance range finder is a safe class II laser device that can measure both near and far. Technical specifications of the sensor are given in Table 1 and FLS C-10 point laser range sensor is given in Fig. 2(a).

TABLE I. TECHNICAL SPECIFICATIONS OF FLS C-10POINT LASER

\begin{tabular}{|c|c|}
\hline \multicolumn{2}{|c|}{ Technical Specifications } \\
\hline Measuring Range & $0.05 \mathrm{~m}-65 \mathrm{~m}$ \\
\hline Accuracy & $\pm 1.0 \mathrm{~mm}$ \\
\hline Max. Measuring Rate & $200 \mathrm{~Hz}$ \\
\hline Dimensions & $150 \times 80 \times 55 \mathrm{~mm}$ \\
\hline Weight & $690 \mathrm{~g}$ \\
\hline Serial interfaces & RS-232, RS-422 \\
\hline Signal Measurement & Single or Continuous \\
\hline
\end{tabular}

Dimetix FLS-C10 sensor is working with phase shift principle. The sensor scans the scene with a periodic light wave and distance to the scene is calculated from the difference between the phase and the frequency of the received signal. Because of no waiting for the received signal, this method provides high sample density and accuracy in comparison with time of flight principle.

\section{B. Line-laser based scanner}

Hokuyo UTM-30LX is a Class I laser distance measurement sensor for many applications that require high speed and accuracy. The sensor can be changed view direction by a rotating mirror and thus measure the points along a line at a time. Hokuyo UTM-30 LX is working with the time of flight principle. This principle is based on the calculation of the time between the transmitted and received laser signals as a result of surface reflection. The distance of the desired point is calculated from speed of light and round trip delay of the signal. UTM-30LX line laser sensor has a rotating mirror and position encoder. With the rotating mirror scanning process is performed along a line and position encoder determines the measurement resolution on the line. The standard encoder resolution is 1080 counts per revolution.

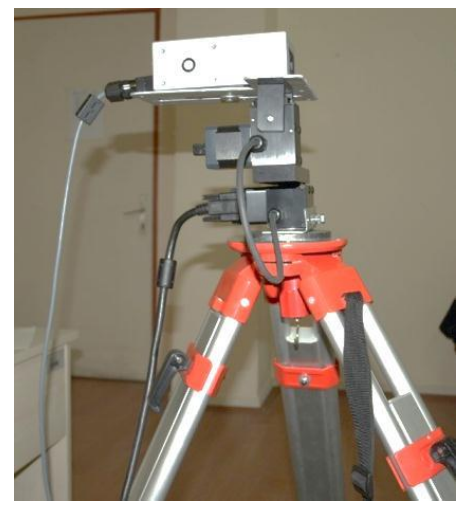

(a)

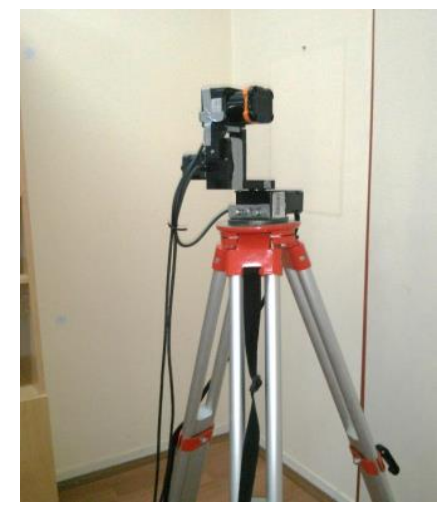

(b)
Fig. 2. Proposed laser scanner systems: (a) point laser, (b) line laser.

TABLE II. HOKUYO UTM-30LX TABLE 1. TECHNICAL SPECIFICATIONS OF HOKUYO UTM-30LX

\begin{tabular}{|c|l|}
\hline \multicolumn{2}{|c|}{ Technical Specifications } \\
\hline Measuring Range & $0.1 \sim 30 \mathrm{~m}$ \\
\hline Accuracy & $0.1-10 \mathrm{~m}: \pm 30 \mathrm{~mm}, 10-30 \mathrm{~m}: \pm 50 \mathrm{~mm}$ \\
\hline Max. Measuring Rate & $40 \mathrm{~Hz}$. \\
\hline View & $270^{\circ}$ \\
\hline Dimensions & $60 \times 60 \times 87 \mathrm{~mm}$ \\
\hline Weight & $210 \mathrm{gr}$ \\
\hline Serial interfaces & RS-232, RS-422 \\
\hline Signal Measurement & Single or Continuous \\
\hline
\end{tabular}

Line laser range finder is mounted up to the pan tilt unit in our system as shown in Fig. 2(b). However, tilt motion is performed by the rotating mirror. Only pan motion can be controlled by the used in this type of scanner. 


\section{3D Data Acquisition System}

Both in point and line laser based scanners, the output is pan angle, tilt angle and distance. The analytical 3D model is given in Fig. 3. In Fig. 3, origin of the coordinate system is intersect of the two axes of control platform $\mathrm{X}$ and $\mathrm{Y}$. $\mathrm{Z}$ axis is parallel to laser distance measuring line. $\mathrm{M}(\mathrm{x}, \mathrm{y}, \mathrm{z})$ is the measured point and $\mathrm{D}$ is the distance between point and control platform. $t$ (tilt angle) is the vertical scanning step angle of control platform and the $\mathrm{p}$ (pan angle) horizontal scanning step angle of control platform. 3D coordinates of the measured point can be calculated from pan angle, tilt angle and distance by Equation 1.

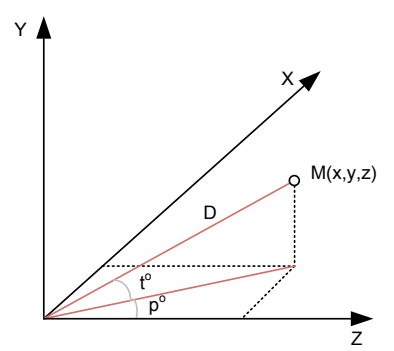

Fig. 3. Analytical 3D model

$$
\begin{aligned}
& y=D \cdot \sin (t) \\
& x=D \cdot \cos (t) \cdot \sin (p) \\
& z=D \cdot \cos (t) \cdot \cos (p)
\end{aligned}
$$

Pan-tilt unit and laser sensors can be controlled from serial port. Developed 3D data acquisition system, provide the control of the pan-tilt unit and laser sensors, according to the user specified parameters. Data acquisition system contains a variety of programs that developed in MATLAB environment to receive, record and process of the pan angle, tilt angle and distance data. In 3D data acquisition systems, user can control scanner system with the parameters of scanning range, step size for pan and tilt, start and finish positions of the pan and tilt. Operation steps of the system can be seen in Fig. 4.

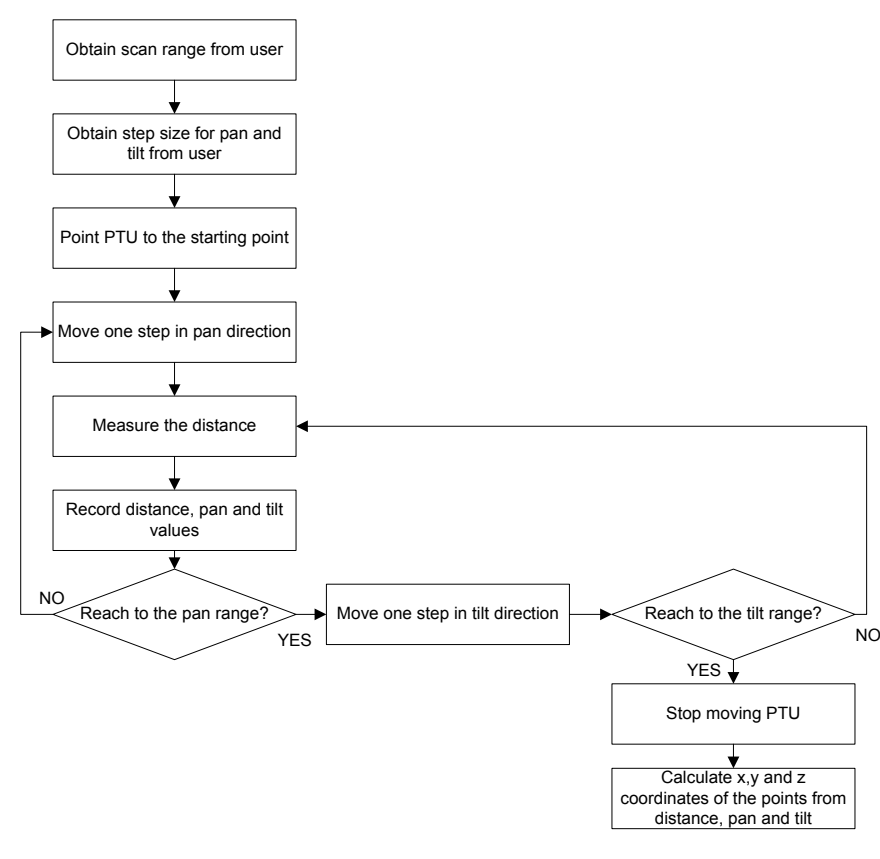

Fig. 4. Flowchart of laser scanner 3D data acquisition software

\section{3D MEASUREMENTS WITH PROPOSED LASER SCANNERS}

Two different laser sensors are used in our system. Point laser can measure a single point at the view of the direction. Localization in horizontal and vertical direction is performed by pan tilt unit. On the other hand, line laser can measure along a line at the view of the direction. Vertical position derived from rotating mirror and position encoder in its structure. Horizontal localization is performed by pan tilt unit. In point laser scanner both in horizontal and vertical positioning depends on the pan tilt unit resolution.

Advantages and disadvantages of both scanner systems are listed below:

- $\quad$ Point laser scanner can measure a single point; on the other hand, line laser scanner can measure the points along a line at a time.

- Point laser scanner is more precise because of the positioning procedure with pan tilt unit both in horizontal and vertical. Line laser sensor has a same resolution in horizontal direction; however the positioning resolution depends on encoder resolution.

- Point laser scanner can capture 20 point in a second. Pan tilt unit resolution is $0,012858^{\circ}$ and this unit can get 24836 different positions for pan and 5823 different position for tilt.

- Line laser has a rotating mirror and 1080 count per revolution. With $25 \mathrm{~Hz}$ measuring rate, it can capture 27000 point at a time. Only pan motion can be 
controlled by user. Pan angle can get 24836 different values.

- Point laser scanner is suitable for applications that details are very important. Scanning time is reasonable for small object measurements.

- Line laser scanner is suitable for applications that require less detail. Scanning time is reasonable for large objects or environments. In particular, applications such as cultural heritage, environment reproduction, indoor and outdoor mapping where the scanning area is large.

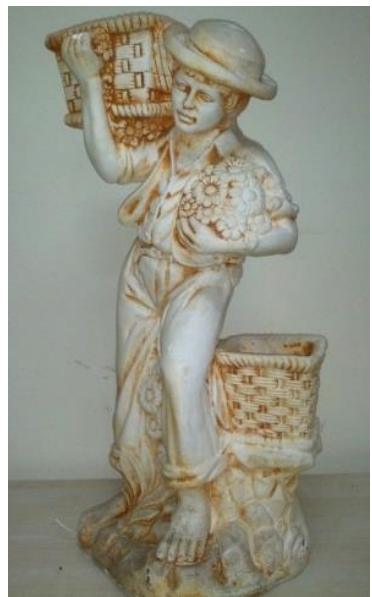

(a)

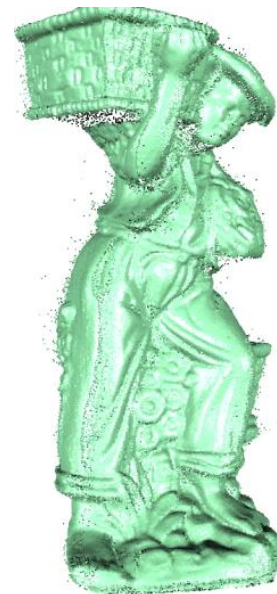

(b)
For the assessment of scanning performances a small sculpture is modeled with point laser scanner and a laboratory is modeled with line laser scanner. 3D point clouds belong to same object or environment taken from different locations laser scanners are registered with well-known Iterative Closest Point algorithm. After registration, obtained noisy point cloud that represents the whole object, is filtered by singular value decomposition method. Obtained point clouds are given in Fig. 5 and 6 for sculpture and laboratory model, respectively.

Fig. 5. Sculpture model: (a) original model, (b) 3D point cloud, (c) noisy mesh, (d) filtered mesh.

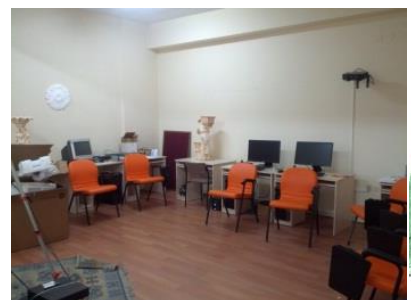

(a)

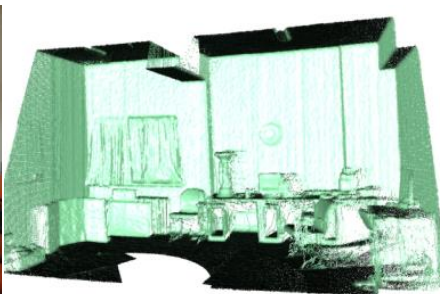

(b)

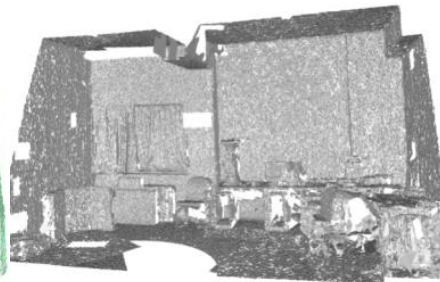

(c)

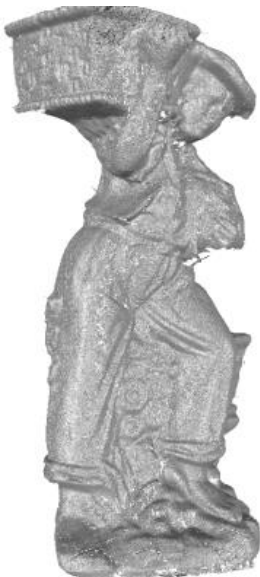

(d)

Fig. 6. Laboratory model: (a) original model, (b) 3D point cloud, (c) noisy mesh, (d) filtered mesh

\section{CONCLUSION}

In this study, two different laser scanning systems are proposed. Scanner systems are differing in terms of laser range finder type. Measurement results showed that point laser based scanner is slower than line laser based scanner. Point laser range finder scanned the sculpture very detailed and scanning time is acceptable. The sculpture is a small object and scanning area is small. Point laser based scanner provides more detailed and more sensitive for scanning small objects and in applications when the details is very important.
Scanning of the laboratory is very easy with line laser and scanning time is very short. This type of sensors can be preferred applications such as cultural heritage, built environment, indoor mapping etc.

\section{ACKNOWLEDGEMENT}

The studies in this paper have been supported by Erciyes University FBA-10-3067, FBA-9-1131.

\section{REFERENCESS}

[1] T. Al-Hawari, et al., "2D laser scanner selection using fuzzy logic," Expert Systems with Applications, vol. 38. 2011, pp. 5614-5619. 
[2] Z. Xie, S. Xu, and X. Li, "A high-accuracy method for fine registration of overlapping point clouds," Image and Vision Computing, vol. 28. 2010, pp. 563-570.

[3] Y. Arayici, "An approach for real world data modelling with the 3D terrestrial laser scanner for built environment," Automation in Construction, vol. 16. 2007, pp. 816-829.

[4] M. Mahmud, et al., "3D part inspection path planning of a laser scanner with control on the uncertainty," Computer-Aided Design, vol. 43. 2011, pp. 345-355.

[5] S. Son, H. Park, and K. H. Lee, "Automated laser scanning system for reverse engineering and inspection," International Journal of Machine Tools and Manufacture, vol. 42. 2002, pp. 889897.

[6] M. Korosec, J. Duhovnik, and N. Vukasinovic, "Identification and optimization of key process parameters in noncontact laser scanning for reverse engineering," Computer-Aided Design, vol. 42. 2010, pp. 744-748.

[7] S. Larsson and J. A. P. Kjellander, "Path planning for laser scanning with an industrial robot," Robotics and Autonomous Systems, vol. 56. 2008, pp. 615-624.

[8] S. Larsson and J. A. P. Kjellander, "Motion control and data capturing for laser scanning with an industrial robot," Robotics and Autonomous Systems, vol. 54. 2006, pp. 453-460.

[9] G. L. Heritage and D. J. Milan, "Terrestrial laser scanning of grain roughness in a gravel-bed river," Geomorphology, vol. 113. 2009, pp. 4-11.

[10] C. Portalés, J. L. Lerma, and S. Navarro, "Augmented reality and photogrammetry: A synergy to visualize physical and virtual city environments," ISPRS Journal of Photogrammetry and Remote Sensing, vol. 65. 2010, pp. 134-142.

[11] D. Miyazaki, et al., "The great buddha project: Modeling cultural heritage through observation," in Modeling from Reality. vol. 640, K. Ikeuchi and Y. Sato, Eds., ed: Springer US, 2001, pp. 181-193.

[12] N. Yastikli, "Documentation of cultural heritage using digital photogrammetry and laser scanning," Journal of Cultural Heritage, vol. 8. 2007, pp. 423427.

[13] K. Schwenzer-Zimmerer, et al., "Quantitative 3D soft tissue analysis of symmetry prior to and after unilateral cleft lip repair compared with non-cleft persons (performed in Cambodia)," Journal of Cranio-Maxillofacial Surgery, vol. 36. 2008, pp. 431-438.

[14] G. Sithole and G. Vosselman, "Experimental comparison of filter algorithms for bare-Earth extraction from airborne laser scanning point clouds," ISPRS Journal of Photogrammetry and Remote Sensing, vol. 59. 2004, pp. 85-101.

[15] S. Izadi, et al., "KinectFusion: real-time dynamic 3D surface reconstruction and interaction," presented at the ACM SIGGRAPH 2011 Talks, Vancouver, British Columbia, Canada, 2011.

[16] D. Samaras, et al., "Variable albedo surface reconstruction from stereo and shape from shading," presented at the IEEE Conference on Computer Vision and Pattern Recognition, South Carolina, USA, 2000.

[17] K. A. Hashim, et al., "Integration of low altitude aerial \& terrestrial photogrammetry data in 3D heritage building modeling," presented at the Control and System Graduate Research Colloquium (ICSGRC), 2012 IEEE, 2012.

[18] P. Kasprzak and P. Kowalczuk, "Objects recognition with high-resolution in SAR data and global geometric feature map," in Signal Processing Symposium (SPS), 2013, 2013, pp. 1-5.

[19] J. J. Atick, P. A. Griffin, and A. N. Redlich, "Statistical approach to shape from shading: reconstruction of three-dimensional face surfaces from single two-dimensional images," Neural Computation, vol. 8. 1996/08/15 1996, pp. 13211340.

[20] G. Pavlidis, et al., "Methods for 3D digitization of cultural heritage," Journal of Cultural Heritage, vol. 8. 2007, pp. 93-98.

[21] FLIR. (2014, 01.06.2014). PTU D46-70 pan-tilt. Available:

http://www.flir.com/mcs/view/?id=53712

[22] Dimetix. (2014, 01.06.2014). FLS-C10 Lazer. Available: http://www.dimetixusa.com/catalog/product_info.php?cPath=22_43\&p roducts id $=88$

[23] Hokuyo. (2014, 01.06.2014). UTM-30LX Lazer. Available: https://www.hokuyoaut.jp/02sensor/07scanner/utm_301x.html 